\title{
Fracture of the Posterior Process of Talus with Pilon Fracture: A case report
}

\author{
by SS Suresh MS Orth, MCh Orth $^{1 ⿴}$
}

The Foot and Ankle Online Journal 3 (12): 1

Fractures of the posterior process of the talus are extremely rare and more so when it is associated with a pilon fracture. Anatomical reduction and fixation of these injuries are important to prevent post traumatic ankle and subtalar arthritis and nonunion. The author presents a case of fracture of the posterior process of the talus with pilon fracture. In addition to these he also had ipsilateral, undisplaced extra articular fracture of the calcaneum, undisplaced fracture of the navicular and fracture of the first metatarsal.

Key words: Talus; Posterior process; Foot; Fracture.

Published: December, 2010

This is an Open Access article distributed under the terms of the Creative Commons Attribution License. It permits unrestricted use, distribution, and reproduction in any medium, provided the original work is properly cited. @The Foot and Ankle Online Journal (www.faoj.org)

$\mathrm{T}$ The posterior process of the talus has two tubercles, the medial and lateral, with the groove for the flexor hallucis longus tendon in between. The more prominent of these is the lateral tubercle. The posterior process is an intra articular component to the subtalar and ankle joints and any incongruity to the posterior process can lead to early degenerative arthritis of both joints.

Fracture of the entire posterior process of the talus is a very rare injury with only 13 cases reported to date. ${ }^{1,2,3,4}$ Pilon fractures of the tibia account for 1 $10 \%$ of lower limb fractures ${ }^{5}$, and is usually the result of high energy trauma in active people. Concomitant injuries reported with posterior process fracture include medial malleolus fracture ${ }^{1}$, and subtalar dislocation $^{6}$. Pilon fracture in association with fracture of entire posterior process of the talus is not reported so far. The patient in this study also had ipsilateral extra articular os calcis fracture, fracture of the navicular and fracture of the first metatarsal bone.

\footnotetext{
Address correspondence to: SS Suresh MS Orth, MCh Orth, PO Box 396, Ibri 516, Oman email: dr.s.s.suresh@gmail.com

${ }^{1}$ Head of Department of Orthopaedics, Department of Orthopaedics, Ibri Regional Referral Hospital, PO Box 46 Ibri 516, Sultanate of Oman.
}

\section{Case Report}

A 29 year-old male expatriate worker was brought to the accident and emergency with history of a heavy sheet falling on his both lower legs. The right ankle and lower leg was grossly swollen. There was mild swelling of the left ankle with tenderness over the lateral malleolus. He didn't have any co-morbid medical illness. He also had a puncture wound over the medial malleolus on the right. There was no distal neurovascular deficit.

X-rays performed in the emergency department showed comminuted fracture of the right distal tibia extending to the tibial plafond and the medial malleolus, with suprasyndesmotic fracture of the fibula. (Figs. $1 \mathrm{~A}$ and B, and 2) In addition, he so had undisplaced fracture of the right os calcis, navicular and first metatarsal bone. X-rays of the left ankle showed an undisplaced fracture of the lateral malleolus. 


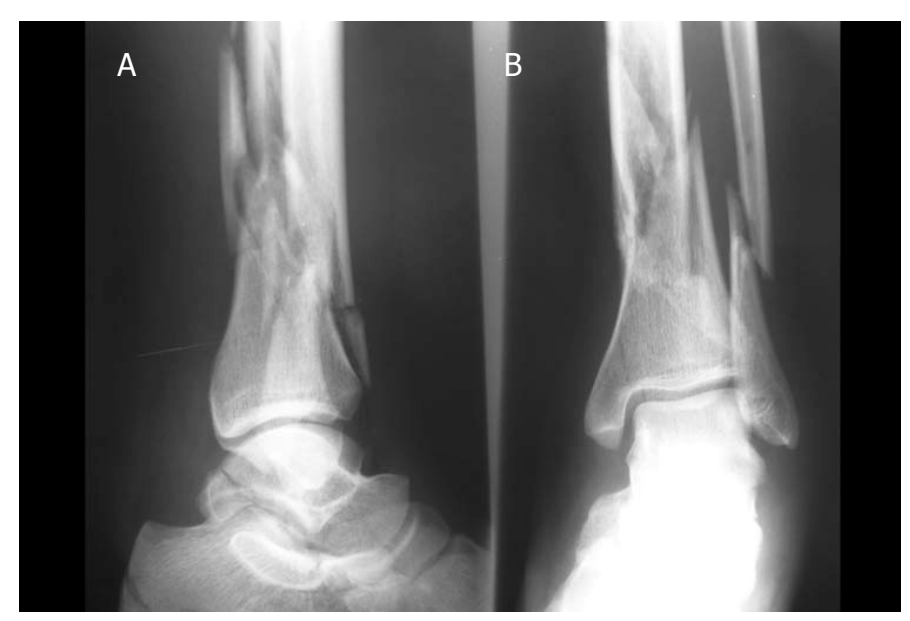

Figure 1 A and B Lateral (A) and anterior posterior (B) view $\mathrm{x}$-rays of the ankle showing the extent of injury.

The puncture wound over the right medial malleolus was debrided in the emergency department operating room, and a back slab was given for comfort. Antibiotic prophylaxis was started with intravenous cephradine due to fear of infection on the medial side. Computed tomography (CT) scan of the right ankle showed minimally displaced fracture of the posterior process of the talus without significant comminution. (Fig. 3)

He was taken for surgery the very next day. Under epidural anaesthesia the fibular fracture was fixed first with a third tubular plate. The wound over the medial malleolus was debrided again and the pilon fracture was fixed through a minimally invasive approach, with stab incisions, and a spoon plate was used for stabilization. The fixation was checked with imaging intra operatively and the posterior process fracture was found minimally displaced. This was approached by extending the lateral incision and fixation was done with a $4 \mathrm{~mm}$ partially threaded cancellous screw. The peroneal tendons were displaced to visualize the fracture.

The soft tissue attachments to the fragment were not disturbed. The wound was closed in layers with a drain on the lateral side. Post operatively the leg was immobilized in a slab for 6 weeks. The patient remained in the hospital for 11 days due to necrosis of skin on the lateral side which eventually healed with dressings.

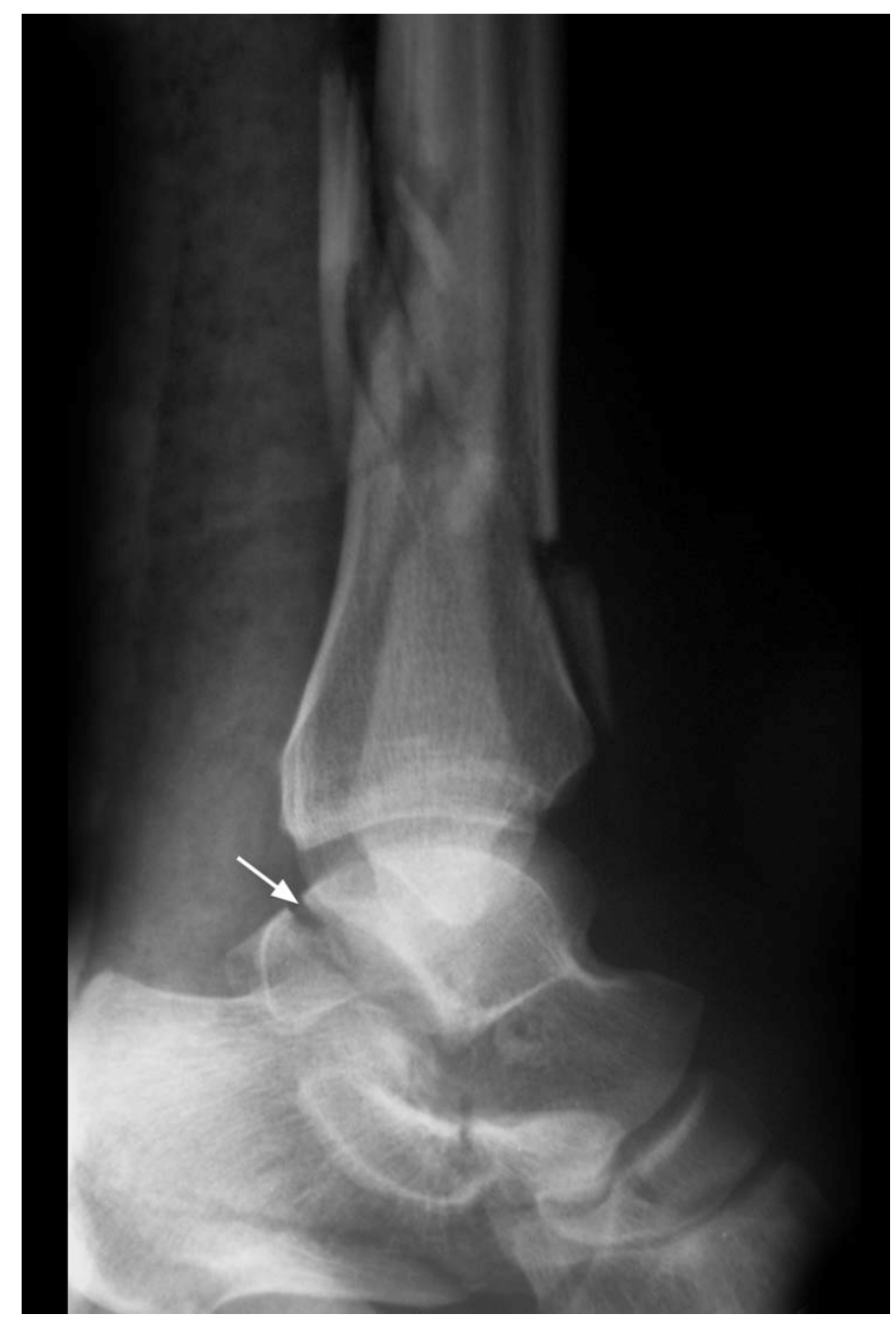

Figure 2 Lateral view of ankle showing posterior process fracture. (see arrow)

$\mathrm{X}$-rays taken during the post operative period showed acceptable reduction of all fractures. (Figs. 4) The patient was followed up at 6 weeks where the immobilization was removed. The patient was also advised non weight bearing mobilization of the ankles. We were unable to follow up this patient as went to his home country and attempts to contact him failed. The patient was informed that data regarding his case would be used for publication and gave his written consent. 

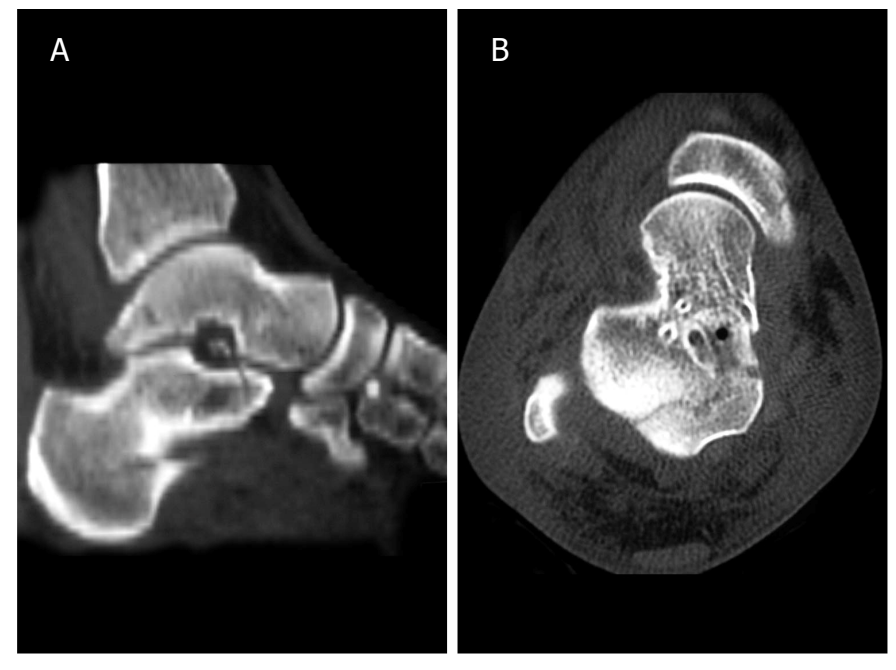

Figure $\mathbf{3 A}$ and $\mathbf{3 B}$ Computed tomography (CT) scan showing posterior process fracture. (A) Fractured posterior talar process on axial CT. (B)

\section{Discussion}

Fractures of the posterior process of the talus are very rare injuries with only few published reports in the English literature. ${ }^{1,3,7}$ Fracture of the posterior process of the talus involves both medial and lateral tubercles There are few reports of isolated fracture of the posterior process after the first report by Nasser and Manoli. ${ }^{4,8,9}$ Subsequent to this reports of posterior process fracture with other ipsilateral injuries have been reported.

The posterior process of the talus has two tubercles, medial and lateral, with the groove for flexor hallucis longus in between. Lateral tubercle is the larger one and this projects more posterior than the medial tubercle. ${ }^{4}$ The fractures of the posterior process cause damage to two joints; the posterior facet of the subtalar joint and the ankle joint. Forced plantar flexion of the foot compressing the posterior talus between the calcaneum and tibia is presumed to be the mechanism of injury. ${ }^{2,3,8}$ Another mechanism documented is forceful inversion of the foot. ${ }^{9}$
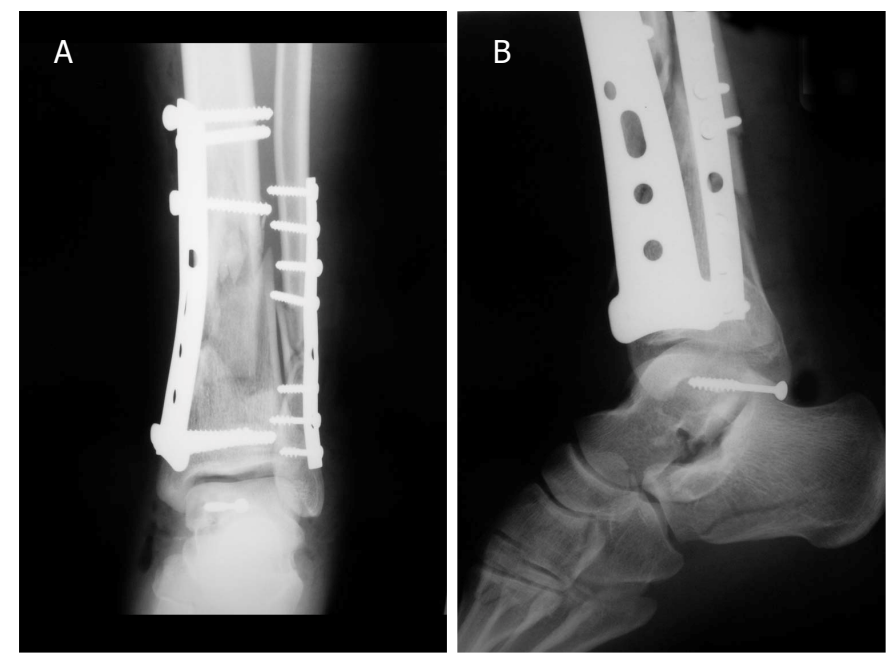

Figure 4A and 4B $X$-rays showing fixation of the fractures. (A) $X$-rays Oblique view of ankle showing reduction of the posterior process fracture. (B)

Prompt diagnosis and appropriate reduction and internal fixation are needed to prevent complications of malunion; non union and post traumatic arthritis. ${ }^{6}$ Management varies from conservative treatment ${ }^{10}$ to immediate open reduction. ${ }^{6}$

Anatomical reduction is important to prevent avascular necrosis and also helps in early mobilization. Moreover presence of an os trigonum can add to the confusion. ${ }^{4,11}$ Failure to diagnose undisplaced fracture can cause painful non-union and significant disability. ${ }^{2,412}$ The commonest fracture in the posterior aspect of the talus is fracture of the lateral tubercle. Lateral tubercle fractures are often missed and misdiagnosed as ankle sprains. ${ }^{12}$

Posteromedial approach, ${ }^{9}$ after mobilization of the neurovascular bundle is used by most of the authors. This fracture can be approached either through a posterolateral or posteromedial approach.' Nadim recommends open reduction if the displacement of the fragment is more than $3 \mathrm{~mm} .{ }^{1,9}$ Though conservative treatment is recommended for minimally displaced or undisplaced fractures the patients can have poor outcomes with persisting painful limitation of ankle movements and recurrent effusion. ${ }^{12}$ 
A CT scan can show the amount of displacement and the degree of comminution. ${ }^{12}$ Reduction and stabilization of the fracture through a posteromedial incision is with risk of damage to the neurovascular structures. Flexor hallucis longus tendon may prevent accurate reduction by closed methods. ${ }^{6}$ In the series by Bhanot, et al., one case was fixed through a separate postero lateral incision though the fracture was visualized and reduced through the medial approach. ${ }^{1}$ In the case report by Naranja the fracture was approached through the posterolateral route. ${ }^{6}$ The soft tissue attachments (insertions of the posterior talofibular ligament and the deltoid ligament) should be carefully preserved during surgery.

Excision of the posterior part of the talus is suggested by Nyska in late diagnosed cases, though there are no published reports. ${ }^{12}$ Concomitant fractures are not reported $^{6,7}$, and to the author's knowledge the only report is that of medial malleolus and posterior process fracture by Bhanot, et al.,. ${ }^{1}$ Naranja, et al., reported on a case of open medial subtalar dislocation in association with fracture of posterior process of the talus. $^{6}$

\section{Conclusions}

High index of suspicion is needed to diagnose isolated posterior process fracture, but if associated with concomitant injuries the diagnosis becomes easy. A CT scan is useful to determine the amount of displacement. Early operative intervention and fixation prevents post traumatic arthritis.

\section{References}

1. Bhanot A, Kaushal R, Bhan R, Gupta PN, Gupta RK, Bahadur R. Fracture of the posterior process of talus. Injury 2004; 35 : 1341-1344.

2. Ahmad R, Ahmad SMY. Fracture of the posterior process of the talus: An unusual injury. Emerg Med J 2007: 24: 867.

3. Prasad G, Mittal D, Harlekar V, Raut VV. Fracture of the posterior process of the talus: A case report. Eur J Orthop Surg Traumatol 2007; 17: 417-419.

4. Nakai T, Murao R, Temporin K, Kakiuchi M. Painful nonunion of fracture of the entire posterior process of the talus: a case report. Arch Orthop Trauma Surg 2005; 125(10) 721-724. 5. Sirkin M, Sanders R. The treatment of Pilon fractures. Orthop Clin North Am 2001; 32(1): 91-102.

6. Naranja RJ Jr, Monaghan BA, Okereke E, Williams GR Jr. Case report: Open medial subtalar dislocation associated with fracture of the posterior process of the talus. J Orthop Trauma 1996; 10(2): 142-144.

7. Chen YJ, Liang SC, Huang TJ. Fracture of entire posterior process as an obstacle to reduction of an anterior talar subluxation: Case report. J Trauma 1997; 42(2): 314-317.

8. Nasser S, Manoli A. Fracture of the entire posterior process of the talus: a case report. Foot Ankle 1990; 10(4): 235-238.

9. Nadim Y, Tosic A, Ebraheim N. Open reduction and internal fixation of fracture of the posterior process of the talus: A case report with review of the literature. Foot Ankle Int 1999; 20(1): 50-52.

10. Jimulia TR, Parekh AN. Fracture of the entire posterior process of the talus. J Postgrad Med 1995; 41: 54-55.

11. Kose O, Okan AN, Durakbase MO et al. Fracture of the os trigonum: a case report. J Orthop Surg 2006; 14(3): 354-356.

12. Nyska M, Howard CB, Matan Y, Cohen D, Peyser A, Garti A, Bar-Ziv J. Fracture of the posterior body of the talus-the hidden fracture. Arch Orthop Trauma Surg 1998; 117: 114-117. 